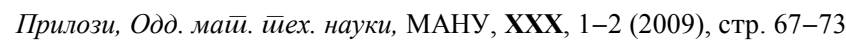
Contributions, Sec. Math. Tech. Sci., MANU, XXX, 1-2 (2009), pp. 67-73

ISSN 0351-3246

UDC: 517.819 .3

Original scientific paper

\title{
GENERALIZATION OF A SUMMATION DUE TO RAMANUJAN
}

\section{Tibor K. Pogány, Arjun K. Rathie, Ujjawal Pandey}

A b s tra ct: The aim of this research note is to find the sum of the series

$$
1+\frac{x-1}{x+1+j}+\frac{(x-1)(x-2)}{(x+1+j)(x+2+j)}+\ldots \quad(\Re\{x\}>0)
$$

for $j=0,1,2,3,4,5$. When $j=0$, we get a summation due to Ramanujan. The results are derived with the help of generalized Kummer's theorem obtained already by Lavoie, Grandie and Rathie.

2000 Mathematics Subject Classification. Primary 33C05; Secondary 33B15, 40A99

Key words and Phrases: Hypergeometric ${ }_{2} F_{1}$; Kummer's summation theorem; Ramanujan summation formula

\section{INTRODUCTION}

We start with an interesting summation due to Ramanujan [4], viz.

$$
1+\frac{x-1}{x+1}+\frac{(x-1)(x-2)}{(x+1)(x+2)}+\ldots=\frac{2^{2 x-1} \Gamma^{2}(x+1)}{\Gamma(2 x+1)} \quad(\mathfrak{R}\{x\}>0) .
$$

As pointed out by Berndt [2], this summation can be obtained quite simply by employing Kummer's summation theorem [1] viz.

$$
{ }_{2} F_{1}\left[\begin{array}{cc}
a, b \\
1+a-b
\end{array} ;-1\right]=\frac{\sqrt{\pi} \Gamma(1+a-b)}{2^{a} \Gamma\left(1+\frac{a}{2}-b\right) \Gamma\left(\frac{a}{2}+\frac{1}{2}\right)}
$$

by taking $a=1$ and $b=1-x$. 
In 1996 Lavoie, Grandie and Rathie [3] have obtained explicite expressions of

$$
{ }_{2} F_{1}\left[\begin{array}{c}
a, b \\
1+a-b+j
\end{array} \quad ;-1\right]
$$

for $j=0, \pm 1, \pm 2, \pm 3, \pm 4, \pm 5$. The case $j=0$ was presented above in (2) as the Kummer's theorem. However, the following another special cases with nonnegative $j$ will be required in our present investigations:

$$
\begin{gathered}
{ }_{2} F_{1}\left[\begin{array}{c}
a, b \\
1+a-b+j
\end{array} ;-1\right]=\frac{\sqrt{\pi} \Gamma(1+a-b+j) \Gamma(1-b)}{2^{a} \Gamma\left(1-b+\frac{1}{2}(j=|j|)\right)} \\
\times\left[\frac{A_{j}}{\Gamma\left(\frac{a}{2}-b+\frac{j}{2}+1\right) \Gamma\left(\frac{a}{2}+\frac{j}{2}+\frac{1}{2}-\left[\frac{j+1}{2}\right]\right)}\right. \\
\left.+\frac{B_{j}}{\Gamma\left(\frac{a}{2}-b+\frac{j}{2}+\frac{1}{2}\right) \Gamma\left(\frac{a}{2}+\frac{1}{2}-\left[\frac{j}{2}\right]\right)}\right]
\end{gathered}
$$

where, as usual, $[x]$ denotes the greatest integer less then or equal to $x$ and the values of the constants $A_{j}, B_{j}$ are given in the Table 1. below.

T a b le 1

\begin{tabular}{|c|c|c|}
\hline$j$ & $A_{j}$ & $B_{j}$ \\
\hline 0 & 1 & 0 \\
\hline 1 & -1 & 1 \\
\hline 2 & $a-b+1$ & -2 \\
\hline 3 & $3 b-2 a-5$ & $2 a-b+1$ \\
\hline 4 & $2(a-b+3)(1+a-b)-(b-1)(b-4)$ & $-4(a-b+2)$ \\
\hline 5 & $\begin{array}{c}-4(6+a-b)^{2}+2(b+11) \\
\times(6+a-b)+b^{2}-13 b-20\end{array}$ & $\begin{array}{c}4(6+a-b)^{2}+2(b-17) \\
\times(6+a-b)-b^{2}-b+62\end{array}$ \\
\hline
\end{tabular}


The aim of this note is to find an interesting generalization of the Ramanujan's summation (1) by making sense of (4). Five summations closely related to Ramanujan's summation have also been obtained as special cases of our main findings. The summations derived here are simple, interesting easily established and may be useful.

\section{MAIN SUMMATION}

\section{Theorem 1.}

$$
\begin{aligned}
1+\frac{x-1}{x+1+j}+ & \frac{(x-1)(x-2)}{(x+1+j)(x+2+j)}+\ldots \\
= & \frac{\sqrt{\pi}(1+j) \Gamma(x)}{2}\left[\frac{A_{j}}{\Gamma\left(x+\frac{1}{2}+\frac{j}{2}\right) \Gamma\left(1+\frac{j}{2}-\left[\frac{1+j}{2}\right]\right)}\right. \\
& \left.+\frac{\Gamma\left(x+\frac{j}{2}\right) \Gamma\left(\frac{1}{2}+\frac{j}{2}-\left[\frac{j}{2}\right]\right)}{B_{j}}\right]
\end{aligned}
$$

for $j=0,1,2,3,4,5$. The coefficients $A_{j}, B_{j}$ can be obtained from the table by changing $a$ by 1 and $b$ by $1-x$ respectively.

Proof. In (4) taking $a=1$ and $b=1-x$, then expressing the hypergeometric function as a series, we have

$$
\begin{aligned}
{ }_{2} F_{1}\left[\begin{array}{ll}
1,1-x \\
1+x+j
\end{array} ;-1\right] & =\sum_{n=0}^{\infty} \frac{(1)_{n}(1-x)_{n}}{(1+x+j)_{n}} \frac{(-1)^{n}}{n !} \\
& =1+\frac{x-1}{x+1+j}+\frac{(x-1)(x-2)}{(x+1+j)(x+2+j)}+\ldots
\end{aligned}
$$


Here $(a)_{0}:=1,(a)_{n}=a(a-1) \ldots(a-n+1), n \in \mathbb{N}$ stands for the Pochhammersymbol, called sometimes shifted factorial as well.

Similarly, putting $a=1, b=1-x$ on the right-hand expression in (4), we get

$$
\begin{array}{r}
\frac{\Gamma\left(\frac{3}{2}\right) \Gamma(x) \Gamma(1+x+j)}{\Gamma(x+j)}\left[\frac{A_{j}}{\Gamma\left(x+\frac{1}{2}+\frac{j}{2}\right) \Gamma\left(1+\frac{j}{2}-\left[\frac{1+j}{2}\right]\right)}\right. \\
\left.+\frac{B_{j}}{\Gamma\left(x+\frac{j}{2}\right) \Gamma\left(\frac{1}{2}+\frac{j}{2}-\left[\frac{j}{2}\right]\right)}\right]
\end{array}
$$

such that, after easy simplification, one tranforms into the asserted right-hand expression of (5). This completes the derivation of (5).

\section{SPECIAL CASES}

In (5), if we take $j=0,1,2,3,4,5$ we have the following interesting summations.

1. For $j=0$

$$
1+\frac{x-1}{x+1}+\frac{(x-1)(x-2)}{(x+1)(x+2)}+\ldots=\frac{\Gamma\left(\frac{3}{2}\right) \Gamma(1+x)}{\Gamma\left(x+\frac{1}{2}\right)}
$$
of (5).

The right-hand side of (8) can be seen equivalent to the right-hand side 
2. For $j=1$

$$
\begin{aligned}
& 1+\frac{x-1}{x+6}+\frac{(x-1)(x-2)}{(x+6)(x+7)}+\ldots \\
& \quad=(1+x) \Gamma(x) \Gamma\left(\frac{3}{2}\right)\left[\frac{1}{\Gamma\left(x+\frac{1}{2}\right)}-\frac{1}{\Gamma\left(\frac{1}{2}\right) \Gamma(x+1)}\right] .
\end{aligned}
$$

3. For $j=2$

$$
\begin{aligned}
& 1+\frac{x-1}{x+3}+\frac{(x-1)(x-2)}{(x+3)(x+4)}+\ldots \\
& \quad=(x+2) \Gamma(x) \Gamma\left(\frac{3}{2}\right)\left[\frac{1+x}{\Gamma\left(x+\frac{3}{2}\right)}-\frac{2}{\Gamma\left(\frac{1}{2}\right) \Gamma(x+1)}\right] .
\end{aligned}
$$

4. For $j=3$

$$
\begin{aligned}
& 1+\frac{x-1}{x+4}+\frac{(x-1)(x-2)}{(x+4)(x+5)}+\ldots \\
& \quad=(x+3) \Gamma(x) \Gamma\left(\frac{3}{2}\right)\left[\frac{x+2}{\Gamma\left(x+\frac{3}{2}\right)}-\frac{3 x+4}{\Gamma\left(\frac{1}{2}\right) \Gamma(x+2)}\right] .
\end{aligned}
$$

\section{For $j=4$}

$$
\begin{aligned}
& 1+\frac{x-1}{x+5}+\frac{(x-1)(x-2)}{(x+5)(x+6)}+\ldots \\
& =(x+2)(x+4) \Gamma(x) \Gamma\left(\frac{3}{2}\right)\left[\frac{x+3}{\Gamma\left(x+\frac{5}{2}\right)}-\frac{4}{\Gamma\left(\frac{1}{2}\right) \Gamma(x+2)}\right] .
\end{aligned}
$$

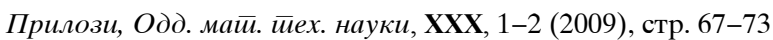


6. For $j=5$

$$
\begin{aligned}
& 1+\frac{x-1}{x+6}+\frac{(x-1)(x-2)}{(x+6)(x+7)}+\ldots \\
& \quad=(x+5) \Gamma(x) \Gamma\left(\frac{3}{2}\right)\left[\frac{x^{2}+7 x+12}{\Gamma\left(x+\frac{5}{2}\right)}-\frac{5 x^{2}+25 x+32}{\Gamma\left(\frac{1}{2}\right) \Gamma(x+3)}\right] .
\end{aligned}
$$

Clearly, (8) is a Ramanujan's summation and other summations (9) to (13) are seen to be closely related (8).

Remark 1. For another summations due to Ramanujan and their generalizations the interested reader can consult [5].

Acknowledgements: The present investigation was supported by the Ministry of Sciences, Education and Sports of Croatia under Research Project No. 112-2352818-2814.

\section{REFERENCES}

[1] W. N. Bailey, Generalized Hypergeometric Series, Cambridge University Press, Cambridge, 1935.

[2] B. C. Berndt, Ramanujan's Notebooks, Part II, Springer-Verlag, New York, 1985.

[3] J. L. Lavoie, F. Grondin, A. K. Rathie, Generalizations of Whipple's theorem on the sum of $\mathrm{a}_{3} F_{2}$, J. Comput. Appl. Math., 72 (1996), 293-300.

[4] S. Ramanujan, Notebooks, Volume 2., Tata Institute of Fundamental Research, Bombay, 1957.

[5] A. K. Rathie, S. Malani, R. Mathur, J. Choi, Certain summations due to Ramanujan and their generalizations, Bull. Korean Math. Soc., 42 (3) (2005), 469-475. 
Р ез и ме

\section{ГЕНЕРАЛИЗАЦИЈА НА СУМИРАњЕТО НА RАМАNUJАN}

Целта на ова истражување е да се најде сумата на редот

$$
1+\frac{x-1}{x+1+j}+\frac{(x-1)(x-2)}{(x+1+j)(x+2+j)}+\ldots \quad(\mathfrak{R}\{x\}>0)
$$

за $j=0,1,2,3,4,5$. За $j=0$, ја добиваме сумата на Ramanujan. Резултатите се добиени со помош на генерализираната теорема на Kummer, дадена од Lavoie, Grandie и Rathie.

Клучни зборови: хипергеометриска ${ }_{2} F_{1}$; сумирање на теоремата на Kummer; сумациона формула на Ramanujan

Address:

\section{Tibor K. Pogány}

Faculty of Maritime Studies, University of Rijeka,

Rijeka, Croatia

E-mail: poganj@pfri.hr

\section{Arjun K. Rathie}

Vedant College of Engineering \& Technology,

TULSI-323 021 (Dist. BUNDI), Rajasthan State, India

E-mail: akrathie@rediffmail.com

\section{Ujjawal Pandey}

Department of Mathematics, Marudhar Engineering College,

Raisar, BIKANER-334 001, Rajasthan State, India

Received: 9. X 2009

Accepted: 1. XII 2009

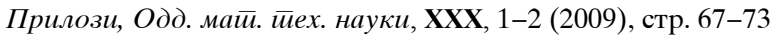

